\title{
Percepciones de estudiantes y docentes sobre las manifestaciones de la violencia escolar en colegios públicos urbanos
}

\author{
Magola del Carmen Quintero-Coronel ${ }^{a *}$ \\ ${ }^{a}$ Magister en Prácticas pedagógicas. Docente Ministerio de Educación Nacional, \\ Secretaría de Educación Municipal de Cúcuta. \\ Forma de citar: Quintero, M.C. (2017). Percepciones de Estudiantes y docentes sobre las \\ manifestaciones de la violencia escolar en colegios públicos urbanos. Perspectivas, 2(1). 70 - 83.
}

Recibido: junio 10 de 2016

Aceptado: septiembre 28 de 2016

\section{Palabras clave \\ Violencia escolar, manifestaciones de la violencia, prevención de la violencia escolar, colegios públicos}

Resumen: La presente investigación tiene como objetivo diagnosticar las manifestaciones de la violencia escolar y las posibles acciones que visualizan estudiantes y docentes para su prevención y atención en los colegios públicos de la zona urbana de Cúcuta. Para evaluar la percepción se utilizó una metodología cuantitativa, con un enfoque descriptivo de carácter exploratorio. Se aplicó una encuesta en escala Likert sistematizada en el software Dyane y la hoja de cálculo Excel. El instrumento se validó mediante el coeficiente de confiabilidad Alfa de Cronbach. La muestra fue de 364 estudiantes, cuyas edades oscilan entre los 10 y 16 años, y 101 docentes de Educación Básica Secundaria. Los resultados de la investigación establecen la existencia de una percepción similar entre educandos y educadores ante el fenómeno de la violencia, coincidiendo en las formas de manifestación, así como también en los lugares desde donde se suscitan estas acciones. Asimismo los participantes plasman su visión acerca de cómo las instituciones educativas pueden implementar estrategias para prevenir y atender las manifestaciones de la violencia escolar.

\footnotetext{
* Autor para correspondencia

madecar12th@hotmail.com
} 


\section{Keywords}

School violence, manifestations of violence, prevention of school violence, public schools

\section{Palavras chave \\ Violência escolar, manifestações de violência, prevenção de violência escolar, escolas públicasinstrumentos de avaliação}

\section{Perceptions of students and teachers about the manifestations of school violence in urban public schools}

\begin{abstract}
The objective of this study is to analyze the manifestations of school violence and the possible responses that students and teachers visualize for its prevention and attention in public schools in the urban area of Cucuta. In order to evaluate the perception, a quantitative methodology coupled with a descriptive approach of an exploratory nature was employed. A Likert scale survey was applied, systematized in the Dyane software and Excel spreadsheet. The instrument was validated using the Cronbach's alpha reliability coefficient. The sample consisted of 364 students, whose ages range between 10 and 16 years, and 101 teachers of Basic Secondary Education. The results of the research establish the existence of a similar perception among students and educators in the face of the phenomenon of violence, coinciding in the forms of manifestation, as well as in the places from which these actions are generated. Likewise, the participants describe their vision of how educational institutions can implement strategies to prevent and address manifestations of school violence.
\end{abstract}

\section{Percepções de estudantes e professores sobre as manifestações de violência escolar nas escolas públicas urbanas}

Resumo: A presente investigação visa diagnosticar as manifestações de violência escolar e as possíveis ações que os alunos e os professores visualizam para sua prevenção e atenção nas escolas públicas da área urbana de Cúcuta. Para avaliar a percepção, utilizou-se uma metodologia quantitativa, com abordagem descritiva de natureza exploratória. Uma pesquisa em escala Likert sistematizada no software Dyane e na planilha Excel foi aplicada. O instrumento foi validado usando o coeficiente de confiabilidade alfa de Cronbach. A amostra foi de 364 alunos, cujas idades variam entre 10 e 16 anos, e 101 professores de Educação Básica e Secundária. Os resultados da pesquisa estabelecem a existência de uma percepção semelhante entre estudantes e educadores diante do fenômeno da violência, coincidindo nas formas de manifestação, bem como nos locais onde essas ações ocorrem. Da mesma forma, os participantes refletem sua visão sobre como as instituições educacionais podem implementar estratégias para prevenir e atender as manifestações da violência escolar. 


\section{Introducción}

La sana convivencia en los espacios educativos se ha visto en tela de juicio durante los últimos años debido al incremento de la violencia en las relaciones interpersonales de estudiantes y docentes; como es natural, esta situación acarrea consecuencias directas en los procesos de enseñanza-aprendizaje.

Para la comprensión de las causas y efectos de este aumento indeseado de la violencia escolar se hacen necesarias investigaciones puntuales que den información sobre esta realidad en las comunidades educativas. En este sentido, Ortega y Mora (1998) se refieren a la violencia escolar como el fenómeno que se hace visible en las instituciones educativas cuando un individuo impone su fuerza, su poder y su habilidad sobre otro, siendo la víctima inocente de cualquier argumento que el violento aporte para justificar su agresión. Otro de los pioneros en investigación sobre violencia en contextos escolares fue Olweus(1998: 25), quien introdujo el término de intimidación escolar y expuso su gravedad en los siguientes términos: "un estudiante es agredido o se convierte en víctima cuando está expuesto de forma repetida y durante un tiempo a acciones negativas que lleva a cabo otro estudiante o varios de ellos".

La violencia escolar se puede manifestar de manera física, verbal, psicológica (cuando se malogra la autoestima y se afianza el temor de los estudiantes) y de manera social (aislamiento del estudiante con el resto del grupo y compañeros). A estas concepciones se une Chaux(2002), quien señala que la agresión y la intimidación son manifestaciones de violencia. Según el autor, la agresión es la acción de hacer daño a otra persona. Es física cuando se maltrata a la persona causando daño corporal; verbal, cuando el maltrato ocurre a través de las palabras ofensivas; y relacional, cuando se ejerce control y perjuicio sobre la vida social de un individuo.

Ante el aumento del fenómeno de la violencia en ámbitos escolares, toda la comunidad educativa debe asumir el reto de proponer estrategias para prevenir, controlar $\mathrm{y}$ atender situaciones de carácter violento. En este sentido se hace necesario identificar las manifestaciones de violencia más habituales que se vivencian en las instituciones educativas.

La percepción de estudiantes y docentes es primordial para obtener esta información dado en los que se entretejen relaciones de interacción y convivencia. Los estudios adelantados hasta el momento señalan que la principal manifestación de violencia entre los estudiantes de secundaria es la agresión verbal. En el caso de violencia contra los docentes, una muy frecuente consiste en el uso de apodos (Oliveros et al, 2009; Hoyos, Aparicio y Córdoba, 2005; Blaya, Derbarbieux, Del Rey y Ortega, 2001; Górriz, 2009).

Cuando se indaga en torno a las estrategias de prevención, se percibe que tanto estudiantes como profesores asumen una visión positiva en cuanto a los esfuerzos realizados por cada institución educativa para mantener la sana convivencia y disminuir los hechos de violencia (Trianes, 2000; Monjas y Avilés, 2006; Díaz y Aguado, 2005; Ortega y Del Rey, 2006). En cuanto a la participación de los docentes en la elaboración de programas de prevención se percibe inconformismo (Paredes, Álvarez, Lega y Vernon, 2008; Ramos, Nieto y Chaux, 2007).

En Colombia, las instituciones educativas cuentan con un Manual de Convivencia, sobre cuya efectividad educadores y estudiantes valoran positivamente en cuanto a las estrategias de prevención. Este instrumento, en efecto, posee normas claras en torno a la violencia y autoriza y orienta a las autoridades competentes de cada institución a la aplicación de procesos efectivos para disminuir hechos violentos que se presenten en los estudiantes. Este Manual es, en este sentido, un instrumento fundamental para mantener la armonía escolar en toda comunidad (Félix, Soriano, Godoy y Martínez, 2008; Puglisi, 2012; Campo, Grisaleña, y Fernández, 2004).

En el contexto anterior, este estudio se ha propuesto describir las percepciones de estudiantes y docentes en torno a las manifestaciones de violencia en contextos escolares en instituciones públicas 
del Municipio de Cúcuta Norte de Santander. Asimismo se pretende caracterizar las formas más recurrentes de violencia que se presentan en estos escenarios con el fin de identificar las diferentes estrategias que estudiantes y docentes consideran para prevenirlas, atenderlas y enfrentarlas dentro de las instituciones educativas.

\section{Materiales y métodos}

El estudio se fundamentó en una metodología de tipo cuantitativo con un enfoque descriptivo y de carácter exploratorio. Con este fin se seleccionó una muestra aleatoria simple de docentes y estudiantes y se confeccionó un cuestionario dirigido a identificar las percepciones de estudiantes y docentes acerca de las distintas formas y manifestaciones de la violencia escolar y de los comportamientos que afectan la sana convivencia en las distintas instituciones educativas públicas del Municipio de San José de Cúcuta. Asimismo se aborda a los sujetos del estudio con respecto a sus valoraciones para prevenir y atender las distintas manifestaciones de violencia escolar.

\subsection{Población y muestra}

La población objeto de estudio está constituida por los docentes y estudiantes de los Colegios Nacional de Comercio, Comuna 1; Santo Ángel, Comuna 2; Pablo Correa León, Comuna 3 y Carlos Pérez Escalante, Comuna 4. Todos pertenecen al ciclo de Educación Básica Secundaria. La muestra participante corresponde a 364 estudiantes cuyas edades oscilan entre edades 10 y 16 años; más los 101 docentes de las cuatro instituciones visitadas. El tamaño de la muestra fue determinado teniendo en cuenta la fórmula diseñada por Hernández et al (1991), con 5\% de error y 95\% de confianza.

Siguiendo un criterio proporcional, la muestra de estudiantes de cada institución se distribuye así: el 35,7\% corresponde al Colegio Pablo Correa León, el 30,8 \% al Colegio Nacional de Comercio, el $17,6 \%$ al Colegio Santo Ángel y el 15,9\% al
Colegio Carlos Pérez Escalante. En cuanto a los docentes encuestados, el porcentaje fue el siguiente: el 51,5\% pertenece al Colegio Pablo Correa León, el 22,8\% al Colegio Santo Ángel y el 12,9\% representa a los Colegios Nacional de Comercio y Carlos Pérez Escalante. En cuanto el género, el 57,4\% de los estudiantes de la población son mujeres y el $42,6 \%$ hombres. En los docentes, la proporción es de $58,4 \%$ de mujeres y $41,6 \%$ de hombres.

\subsection{Instrumentos}

Para recolectar la información se aplicó una encuesta a partir del cuestionario de Buss y Perry (1992), para lo que fue precisa una adaptación previa de algunas de las preguntas con el propósito de fundamentar de manera válida y confiable los resultados obtenidos. A cada variable se asoció una pregunta (con escala tipo Likert), que corresponden a los distintos ítems en las tres subcategorías (prevención, manifestación, atención). A fin de probar la validez del instrumento se realizó un pilotaje a 25 docentes de instituciones públicas, con un grado de confiabilidad de 0,7725, y a 17 estudiantes, con un grado de confiabilidad de Cronbach igual a0.7662.

\subsection{Procedimiento}

Una vez validado y probado el instrumento se procedió a aplicarlo a la muestra seleccionada. Los pasos seguidos fueron estos: aplicación de la encuesta, registro de los datos, análisis del perfil socio demográfico y procesamiento general de la escala de violencia a partir de análisis de tablas de frecuencias y tablas de contingencia. Como software de apoyo al análisis de la información y generación de los resultados se utilizó el programa Dyane y como complemento Microsoft Excel.

\section{Resultados y discusión}

A continuación se presentan un análisis estadístico descriptivo de las variables más 
importantes, lo que permite apreciar y relacionar los aspectos teóricos de interés. Con este fin se presentan tablas de frecuencias considerando como factores eventualmente explicativos el género, la edad y el tipo de participante. Se presentan inicialmente las distintas formas de violencia, los lugares y los mecanismos de prevención.

La tabla 1 recoge los resultados relacionados con la percepción de los estudiantes con respecto a ofensas y/o ridiculizaciones recibidas por los compañeros según el género. De acuerdo con los datos obtenidos, el $65,55 \%$ de las mujeres y el $55,48 \%$ de los hombres niegan haber sido objeto de esta situación en sus establecimientos, pero el $34,45 \%$ de las mujeres y el $44,51 \%$ de hombres manifiestan lo contrario.

Tabla 1

Percepción de los estudiantes de ofensas y/o ridiculizaciones recibidas por los compañeros según el género.

\begin{tabular}{|c|c|c|c|c|c|c|c|}
\hline & \multirow[b]{2}{*}{$\begin{array}{l}\text { ¿Sus compañeros lo } \\
\text { han ofendido y/o } \\
\text { ridiculizado? }\end{array}$} & \multirow{2}{*}{\multicolumn{2}{|c|}{ Total muestra }} & \multicolumn{4}{|c|}{ Género } \\
\hline & & & & \multicolumn{2}{|c|}{ Femenino } & \multicolumn{2}{|c|}{ Masculino } \\
\hline & Valores de la escala & $\mathrm{f}$ & $\%$ & $\mathrm{f}$ & $\%$ & $\mathrm{f}$ & $\%$ \\
\hline 1 & nunca & 144 & 39,56 & 101 & 48,33 & 43 & 27,74 \\
\hline 2 & casi nunca & 79 & 21,70 & 36 & 17,22 & 43 & 27,74 \\
\hline 3 & algunas veces & 115 & 31,59 & 60 & 28,71 & 55 & 35,48 \\
\hline 4 & casi siempre & 17 & 4,67 & 7 & 3,35 & 10 & 6,45 \\
\hline \multirow[t]{2}{*}{5} & siempre & 9 & 2,47 & 5 & 2,39 & 4 & 2,58 \\
\hline & total & 364 & 100,00 & 209 & 100,00 & 155 & 100,00 \\
\hline
\end{tabular}


Lo anterior refleja que las ofensas y/o ridiculizaciones entre estudiantes vienen tomando fuerza en sus relaciones interpersonales. Al indagar entre los docentes si ellos han sido víctimas de esta situación, la opinión del $45,76 \%$ de las mujeres y el $47,72 \%$ de los hombres es la de que este tipo de violencia no está presente entre sus estudiantes (ver tabla 2).

Tabla 2

Percepción de los estudiantes según el género y ofensas y/o ridiculizaciones recibidas por los compañeros

\begin{tabular}{|c|c|c|c|c|c|c|}
\hline \multirow{2}{*}{$\begin{array}{c}\text { ¿Ha recibido agresiones verbales } \\
\text { (ofensas, apodos, insultos, burlas) } \\
\text { de los estudiantes? }\end{array}$} & & & & \multicolumn{3}{|c|}{ Género } \\
\hline & & Total muestra & & Femenino & & Masculin \\
\hline Valores de la escala & $\mathrm{f}$ & $\%$ & $\mathrm{f}$ & $\%$ & $\mathrm{f}$ & $\%$ \\
\hline nunca & 51 & 50,50 & 31 & 52,54 & 20 & $\begin{array}{c}47,6 \\
2\end{array}$ \\
\hline casi nunca & 26 & 25,74 & 14 & 23,73 & 12 & $\begin{array}{c}28,5 \\
7\end{array}$ \\
\hline algunas veces & 21 & 20,79 & 13 & 22,03 & 8 & $\begin{array}{c}19,0 \\
5\end{array}$ \\
\hline casi siempre & 2 & 1,98 & 0 & 0,00 & 2 & 4,76 \\
\hline siempre & 1 & 0,99 & 1 & 1,69 & 0 & 0,00 \\
\hline TOTAL & 101 & 100,00 & 59 & 100,00 & 42 & $\begin{array}{c}100 \\
00 \\
\end{array}$ \\
\hline
\end{tabular}

Fuente: Autor, Proceso de investigación

Las situaciones de violencia escolar tienen su ocurrencia en lugares específicos de la institución. En este sentido, los datos recabados informan sobre los sitios que resultan más proclives (y por tanto, sobre los que se debe ejercer especial vigilancia) para la génesis de actos violentos. Las tablas 3 y 4 muestran las respuestas de los participantes encuestados.

Tabla 3

Percepción de estudiantes según su género sobre los lugares de la institución donde se presentan hechos de violencia

\begin{tabular}{|c|c|c|c|c|c|c|}
\hline \multirow{2}{*}{$\begin{array}{c}\text { Señale los lugares de la Institución } \\
\text { donde se presentan hechos de } \\
\text { violencia }\end{array}$} & & & \multicolumn{4}{|c|}{ Género } \\
\hline & \multicolumn{2}{|c|}{ Total muestra } & & & \multicolumn{2}{|c|}{ Masculino } \\
\hline Lugares & $\mathrm{f}$ & $\%$ & $\mathrm{f}$ & $\%$ & $\mathrm{f}$ & $\%$ \\
\hline Salón de clase & 166 & 45,60 & 101 & 48,33 & 65 & 41,94 \\
\hline
\end{tabular}




\begin{tabular}{|c|c|c|c|c|c|c|c|}
\hline 2 & Patio/cancha & 137 & 37,64 & 76 & 36,36 & 61 & 39,35 \\
\hline 3 & Baños & 34 & 9,34 & 18 & 8,61 & 16 & 10,32 \\
\hline 4 & Caseta/cafetería & 18 & 4,95 & 11 & 5,26 & 7 & 4,52 \\
\hline 5 & Salida del colegio & 251 & 68,96 & 141 & 67,46 & 110 & 70,97 \\
\hline 6 & Ningún lugar & 40 & 10,99 & 25 & 11,96 & 15 & 9,68 \\
\hline & Total & 646 & $(364)$ & 372 & (209) & 274 & $(155)$ \\
\hline
\end{tabular}

Fuente: Autor, Proceso de investigación

Según la percepción de estudiantes en relación con su género, el lugar de mayor incidencia para situaciones violentas es la salida del colegio. Esta opinión está respaldada por el $71 \%$ de los hombres y el $67,5 \%$ de las mujeres. El lugar que continúa en este orden de hechos es el salón de clase, donde es percibida por el $48.3 \%$ de las mujeres y el $41,9 \%$ de los hombres. Le sigue el 39,4\% de hombres y el $36,4 \%$ de mujeres que consideran que el patio de recreo es un lugar propicio para estas acciones por parte de los compañeros.

Tabla 4

Percepción de docentes según su género sobre los lugares de la institución donde se presentan hechos de violencia

\begin{tabular}{|c|c|c|c|c|c|c|c|c|c|c|}
\hline \multicolumn{2}{|c|}{$\begin{array}{l}\text { Señale los lugares de la Institución donde } \\
\text { se presentan hechos de violencia }\end{array}$} & \multirow{2}{*}{\multicolumn{3}{|c|}{ Total muestra }} & \multicolumn{6}{|c|}{ Género } \\
\hline & & & & & \multicolumn{3}{|c|}{ Femenino } & \multicolumn{3}{|c|}{ Masculino } \\
\hline \multicolumn{2}{|r|}{ Lugares } & $\mathrm{f}$ & & $\%$ & $\mathrm{f}$ & & $\%$ & $\mathrm{f}$ & & $\%$ \\
\hline 1 & Salón de clase & & 59 & 58,42 & & 37 & 62,71 & & 22 & 52,38 \\
\hline 2 & Patio/cancha & & 50 & 49,50 & & 26 & 44,07 & & 24 & 57,14 \\
\hline 3 & Baños & & 21 & 20,79 & & 9 & 15,25 & & 12 & 28,57 \\
\hline 4 & Caseta/cafetería & & 10 & 9,90 & & 4 & 6,78 & & 6 & 14,29 \\
\hline 5 & Salida del colegio & & 64 & 63,37 & & 37 & 62,71 & & 27 & 64,29 \\
\hline
\end{tabular}




\begin{tabular}{rrrrrrrr}
6 Ningún lugar & & 11 & 10,89 & 5 & 8,47 & 6 & 14,29 \\
\hline & & & & & & \\
& Total & 215 & $(101)$ & 118 & $(59)$ & 97 & $(42)$ \\
\hline
\end{tabular}

Fuente: Autor, Proceso de investigación

Los docentes, por su parte, poseen una perspectiva similar a la de los estudiantes. Opinan, en efecto, que el lugar de mayor presencia de violencia escolar es la salida del colegio: $64,3 \%$ de los hombres y el 62,7\% las mujeres así lo señalan. En una segunda instancia se percibe que para el $62,71 \%$ de las docentes el salón de clase es escenario de estos hechos, mientras que lo es para el 57,1\% de los profesores opta por el patio de recreo. Le sigue el 52,4\% de hombres que señalan el salón de clase y el $44,1 \%$ de las mujeres que estima que es el patio de recreo. Como se observa, existe percepción cruzada según el género de los docentes. También se pone de manifiesto que en los baños ocurren hechos violentos. Así lo afirma el 28,6\% de hombres y el $15,3 \%$ de las mujeres. Existe una minoría de docentes que se inclinan por la cafetería: el14, 3\% de ellos y el $8.5 \%$ de ellas la señalan. El 14,3\% de los hombres y el 6,8\% de las mujeres, finalmente, niegan situaciones violentas en los espacios de la institución.

En lo que respecta a las estrategias de prevención se encontró que los participantes tienen una visión positiva sobre el manejo de situaciones en las instituciones para disminuir el fenómeno de la violencia escolar (ver tablas 5 y 6 ).

Tabla 5

Valoración de la enseñanza para prevenir la violencia escolar entre estudiantes de acuerdo con su rango de edad

\begin{tabular}{|c|c|c|c|c|c|c|c|c|c|}
\hline \multicolumn{2}{|c|}{$\begin{array}{c}\text { ¿La institución le enseña a } \\
\text { cómo prevenir la violencia } \\
\text { escolar? }\end{array}$} & \multicolumn{2}{|c|}{ Total muestra } & 10-12 años & \multicolumn{5}{|c|}{ Rango de edad } \\
\hline & Valores de la Escala & $\mathrm{f}$ & $\%$ & $\mathrm{f}$ & $\%$ & $\mathrm{f}$ & $\%$ & $\mathrm{f}$ & $\%$ \\
\hline 1 & nunca & 37 & 10,16 & 18 & $\begin{array}{l}14,2 \\
9\end{array}$ & 14 & 7,61 & 5 & 9,80 \\
\hline 2 & casi nunca & 22 & 6,04 & 6 & $\begin{array}{l}4,7 \\
6\end{array}$ & 13 & 7,07 & 3 & 5,88 \\
\hline 3 & algunas veces & 51 & 14,01 & 12 & $\begin{array}{l}9,5 \\
2\end{array}$ & 28 & 15,22 & 10 & 19,61 \\
\hline 4 & casi siempre & 69 & 18,96 & 19 & $\begin{array}{l}15,0 \\
8\end{array}$ & 33 & 17,93 & 16 & 31,37 \\
\hline \multirow[t]{2}{*}{5} & siempre & 185 & 50,82 & 71 & $\begin{array}{l}56,3 \\
5\end{array}$ & 96 & 52,17 & 17 & 33,33 \\
\hline & Total & 364 & 100,00 & 126 & $\begin{array}{l}100,0 \\
0\end{array}$ & 184 & 100,00 & 51 & 100,00 \\
\hline
\end{tabular}


Una proporción importante de estudiantes coincide en que la institución enseña a prevenir la violencia escolar. En efecto, las opciones siempre y casi siempre han sido elegidas con porcentajes superiores a $60 \% .71,4 \%$, de este grupo se encuentra entre $\operatorname{los} 10$ a 12 años, el 70,1\% se encuentra en la categoría de 13 a 15 años y el 64,4\% son los que tienen más de 16 años de edad. Es mínima (alrededor del 10\%) la visión negativa o de duda que manifiestan los estudiantes en referencia a las acciones de prevención promovidas desde la institución.

Tabla 6

Participación de los docentes según su rango de edad en el diseño de estrategias para la prevención de violencia escolar

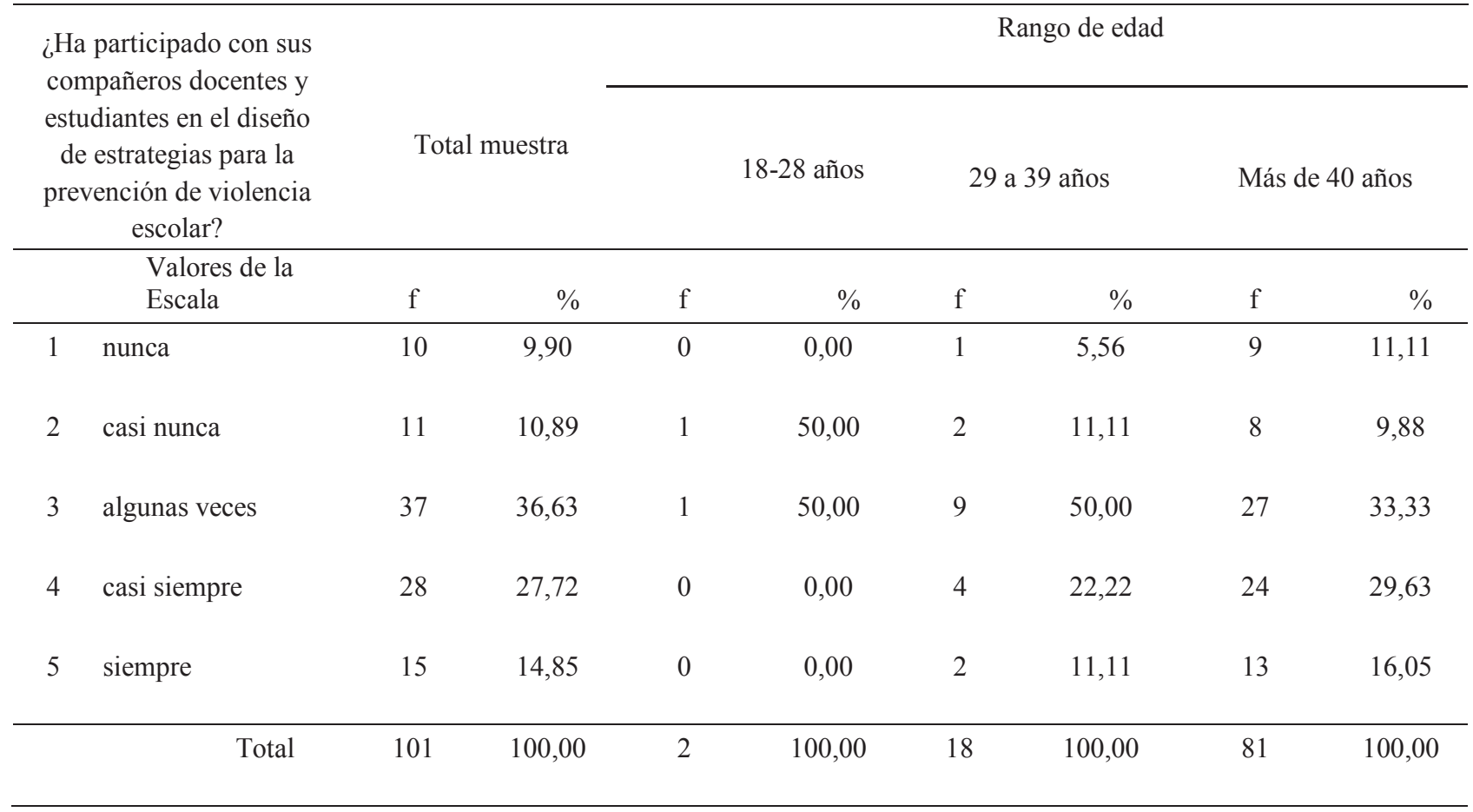

Entre los profesores, el 50\% de los encuestados entre 29 y 39 años expresan haber participado en el diseño de estrategias para la prevención de la violencia en la institución, con lo cual se constituyen en el grupo de edad que lidera estas acciones. El $33,3 \%$ de los mayores de 40 años también validan esta opción, con lo cual confirman su participación, la de compañeros y estudiantes en el diseño de estrategias de prevención.
En el rango de edades que va de los 29 a 39 años se observa una distribución de igual frecuencia para las únicas opciones que superaron el $50 \%$ de las respuestas, con la elección de las alternativas algunas veces y casi nunca. Existe un porcentaje significativo para los mayores de 40 años y de 29 a 39 años que opinan, con sumatorias en porcentajes del $33 \%$ y el $46 \%$, que casi siempre y siempre participan. En estos mismos rangos de edad niegan su participación con porcentajes menores a $22 \%$. 
Con respecto a las formas como se atiende un hecho violento, los estudiantes y docente tienen una percepción similar, tal y como indican los resultados. Los estudiantes (tanto mujeres como hombres, con un $59,8 \%$ y $56,8 \%$ respectivamente) perciben que los docentes atienden oportunamente los hechos violentos. Solo el 18,1 \% de hombres y el 16,3\%de las mujeres muestran inconformismo al respecto.

En lo que concierne a la opinión de los docentes el $86,4 \%$ de las mujeres y el $83,7 \%$ de los hombres confirman que atienden oportunamente cualquier situación de violencia que se presenta entre los estudiantes, mientras el $14,3 \%$ de los hombres y el $11,9 \%$ de las mujeres manifiestan dudas al respecto.

\section{Discusión}

Las percepciones de los estudiantes según el sexo muestran que las mujeres presentan criterios similares frente a las situaciones de violencia, mientras que los hombres presentan mayor desacuerdo, lo cual hace deducir que entre los hombres los actos de violencia son más frecuentes que en las mujeres y que se pueden haber presentado de distintas formas sin que las directivas y los docentes lo hayan detectado. Dicha percepción es abordada por Moral (2005) cuando afirma que los hombres son más permisivos en la tolerancia tanto de la violencia física como la violencia verbal y psicosocial. Domínguez y Manzo (2011) difieren de esta opinión, pues señalan que las mujeres agreden más de forma verbal mientras los hombres lo hacen de forma física. Paredes, Lega, Cabezas, Ortega, Medinay Vega (2010) encuentran en sus investigaciones que ambos géneros participan en comportamientos agresivos frecuentes $y$ en proporciones similares. La postura de los últimos autores se distancia de los hallazgos encontrados en las instituciones participantes

En cuanto a la presencia de actos de violencia escolar en los colegios, la mitad de los estudiantes encuestados señala que en ocasiones se presentan las ofensas. Los resultados de este trabajo coincide con las investigaciones realizadas Oliveros et al.(2009) y
Hoyos, Aparicio y Córdoba (2005), quienes también encuentran que la agresión verbal es la de mayor incidencia. Sin embargo, una parte de la población encuestada piensa que esta situación no se presenta en sus instituciones, y otro grupo, menor, señala que constatan la existencia de ofensas periódicamente. Por tanto, no existe una diferencia marcada entre los que tienen una visión negativa y positiva frente al fenómeno estudiado.

Aunque un alto porcentaje de los estudiantes expresa no ser víctima de ofensas, no deja de ser preocupante el hecho de que un grupo minoritario deje entrever que algunas veces se presenta esta situación. Por otro lado, los estudiantes manifiestan que existen amenazas en los diferentes espacios de las instituciones. En ello, este trabajo coincide con Blaya, Derbarbieux, Del Rey y Ortega (2006) y Górriz (2009), quienes afirman que generalmente los adolescentes presentan actitudes contrarias al empleo de la violencia física, pero se muestran más permisivos ante otras manifestaciones indirectas, como el empleo de la violencia verbal y psicosocial (poner apodos, propagar rumores o exclusión grupal de las víctimas). Los encuestados reconocen que existen ofensas en su plantel y los que confirman la presencia de amenazas, demuestra, como se plantea en la literatura, que la principal manifestación agresiva en los planteles ocurre mediante la asignación de apodos, y que las agresiones verbales se realizan con el ánimo de ofender, intimidar y ridiculizar permanentemente las acciones que las personas realizan al interior del aula de clase o en cualquier otro espacio educativo; que los insultos y las bromas de mal gusto ejecutadas por adolescentes que no sobrepasan los 16 años, tienen como propósito presionar y subestimar las capacidades de las personas ofendidas, y que en este sentido los hombres muestran unas actitudes más permisivas, máxime cuando cursan grados inferiores. La diferencia sustancial entre esta y otras investigaciones tiene que ver con la presencia de un alto porcentaje de estudiantes que manifiestan no haber sido objeto de ofensas ni amenazas. Los autores consultados, en efecto, mencionan 
que si existe violencia en la institución en un alto porcentaje, esta se debe evidenciar en el creciente número de estudiantes con problemas de amenazas y ofensas.

Dentro de los temas contenidos en las preguntas de la encuesta, uno considerado básico para el estudio de la violencia escolar se orientó a indagar sobre los lugares de la institución donde se generan situaciones que la propician. Los estudiantes de los cuatro colegios y también los maestros coincidieron en que la salida de la institución es el espacio que más se presta para que ocurran agresiones físicas, empujones, malas palabras e insultos. La salida del colegio, en efecto, es señalada por el $69 \%$ de los encuestados como espacio para la manifestación de conductas violentas. Más un $30 \%$, por otra parte, ubica el aula de clase como otro espacio generador de violencia. En este sentido, conviene recordar y prestigiar el valioso papel que juega el docente en la formación del estudiante, no solo como ideal de autoridad y conocimiento, sino además como modelo de respeto y tolerancia. Así lo señalan, y con total claridad, Domínguez y Manzo (2011) y Oliveros et al (2009), al manifestar que no existe una mejor forma de evitar que los jóvenes presencian y participen del bullying en los colegios que involucrar a los docentes en los proceso de formación y desarrollo de la convivencia escolar. Este reconocimiento, por otra parte, ya está siendo dado por los estudiantes, pues, el 75,55\% opina que existe una atención oportuna de los docentes en situaciones de violencia escolar. Convendría, pues, aprovechar al máximo el prestigio del docente como mediador en situaciones de violencia para prevenir que estas se sucedan.

En lo que tiene que ver con la prevención y los planes de atención para disuadir a los estudiantes y docentes de practicar la violencia en el ámbito escolar, se percibe negativismo tanto en los unos como los otros. Los estudiantes, en un porcentaje de $69,78 \%$, consideran que aunque la institución se preocupa por formarlos y enseñarles a prevenir cualquier acto de violencia escolar, no han sido tenidos en cuenta para definir y diseñar las estrategias y actividades necesarias para prevenir la violencia escolar en su colegio. Es a lo que se refieren Paredes, Álvarez, Lega y Vernon (2008) cuando en sus investigaciones señalan la escasa participación de los de la comunidad educativa en las estrategias para disminuir situaciones violentas y brindar seguridad a las víctimas.

En esta misma dirección se pronunciaron los docentes, pues aunque participan en la elaboración de programas y controles para evitar la discriminación y la violencia, casi nunca han tenido la oportunidad de presentar propuestas y estrategias de impacto que mejoren la calidad de las relaciones humanas en el colegio. Esta percepción de los educadores se asemeja a la visión que tienen Ramos, Nieto y Chaux (2007), cuando destacan la figura del maestro en los procesos de ayuda en los estudiantes en el ejercicio de competencias ciudadanas para así enfrentar asertivamente hechos de conflicto en las instituciones educativas.

Autores como Carpintero, López, Del Campo, Soriano y Lázaro (2007) y Díaz y Aguado (2005), ratifican el hecho de considerar como una gran falencia de las instituciones en general el hecho de que ni estudiantes ni docentes estén conformes plenamente con las políticas de prevención por parte de las directivas, debido fundamentalmente a la falta de participación y espacios de construcción de propuestas colectivas por parte de toda la comunidad educativa. Según los autores, se trata de variables de personalidad como la de quererse y valorarse como medio para poder querer y valorar a los demás; variables cognitivas, como tener una visión del mundo, de la vida y del ser humano que reconozca que aunque los seres humanos nos comportamos con frecuencia de modo egoísta, tenemos recursos para aprender a trabajar por el bienestar común; o ser capaces de elegir de forma crítica los propios valores y de tener una conducta coherente con ellos. Se trata también de variables afectivas como la empatía y la capacidad para ponernos en sintonía emocional con el otro, comprendiendo lo que siente y teniendo sentimientos "en la misma onda" que los suyos, o el autocontrol. Alumnos y docentes 
en los cuatro colegios objetos de la investigación en Cúcuta, coinciden en considerar que además de los mensajes sobre el tema a través de carteles y de charlas eventuales sobre la importancia de la convivencia y el buen trato en los colegios, y de manuales y libros de seguimiento, se deben integrar en la formulación de las estrategias de choque contra la violencia, las vivencias de los estudiantes y las experiencias de los docentes, para enriquecer las actividades diarias de concientización institucional.

En forma similar para las acciones institucionales, la visión positiva que poseen los encuestados acerca de la atención oportuna de violencia escolar tiene que ver con la funcionalidad y efectividad del manual de convivencia. Las estrategias de prevención que contiene son positivamente valoradas, pues se piensa que favorecen la convivencia escolar. Esta, en efecto, es la percepción de más del $80 \%$ de los participantes encuestados, percepción que coincide con Félix, Soriano, Godoy y Martínez (2008), quienes afirman que un manual de convivencia bien estructurado en conceptos acerca de la violencia, estrategias que fortalecen la convivencia y el compromiso de las autoridades educativas ante su aparición en las instituciones, mantienen sanos ambientes escolares. Sin embargo, existe un porcentaje importante de estudiantes (40\%) que algunas veces cuestiona la funcionalidad de este instrumento de control, no tiene mayor funcionalidad debido a que no se les ha tenido en cuenta para su elaboración. Este hallazgo se apoya en el discurso de Puglisi (2012), quien manifiesta que si el manual se elabora sin la participación de todos los sectores que conforman la comunidad educativa es un instrumento nulo o poco funcional.

En medio de todo, entre estudiantes y maestros se perciben mayores acuerdos que desacuerdos en cuanto a sus respuestas a las preguntas sobre manifestaciones, prevención y atención de la violencia en sus colegios. Entre los acuerdos detectados se puede mencionar el hecho de que los dos colectivos opinen en un porcentaje cercano al 55\%, que los apodos es una de las más frecuentes formas de agresión empleadas en los planteles. Ello confirma la apreciación de Domínguez y Manzo (2011), Oliveros et al. (2009), producto de sus investigaciones. Un45\%de los participantes(sumados docentes y alumnos) también coincide en que el lugar donde se ejerce mayoritariamente alguna clase de violencia es la salida de la institución.

Asimismo coinciden en considerar que en cada colegio ha sido efectiva la utilización oportuna de planes y programas de prevención y atención a fin de evitar la violencia entre los miembros de la comunidad educativa. La diferencia de apreciación se enfoca hacia la existencia de discriminación: los maestros no creen que esté ocurriendo, mientras que los estudiantes manifiestan la presencia, aunque sea en ocasiones, de señalamientos por la apariencia o limitación física, condición social o nivel económico. En términos generales, sin embargo, en los cuatro colegios prevalecen las relaciones diarias de educación y formación, un ambiente favorable para el crecimiento de la convivencia pacífica, la autoestima y la libertad.

\section{Conclusiones}

En los colegios de Cúcuta que fueron objeto de la investigación existe consenso para la negación de la presencia de casos graves de violencia en sus instalaciones. Consideran, en efecto, que los momentos de alteración en el ambiente académico no son constantes sino episódicos, y muchas veces se deben a circunstancias personales que los estudiantes trasladan a las escuelas.

En la población participante (tanto entre estudiantes como docentes) existe unanimidad de criterio en la percepción acerca la principal manifestación del fenómeno de la violencia, que consiste en la adjudicación de apodos. También coinciden, como se ha dicho, en que la salida del colegio es el lugar de mayor presencia de hechos violentos que alteran la convivencia escolar.

Entre la mayoría de los encuestados existe concordancia en cuanto a la utilización de carteles 
y charlas como recursos didácticos de prevención contra la violencia. Las divergencias están dadas fundamentalmente a nivel de colegios como Santo Ángel y el Pablo Correa León, en la forma como estos medios se emplean para evitar hechos graves que alteran el orden institucional; mientras los primeros opinan que son atractivos y que en su diseño ellos son tenidos en cuenta, los segundos manifiestan que hace falta mayor participación de los docentes en la elaboración de estrategias, para erradicar el problema en sus instituciones.

Es claro que el tema de la violencia escolar debe ser una prioridad en la planeación de las estrategias escolares, que los programas y proyectos de estos cuatro colegios en Cúcuta, tienen que enfocarse fundamentalmente hacia el reconocimiento de los valores humanos, sociales, familiares y culturales y que las acciones y los discursos pedagógicos, deben manejar un lenguaje para la convivencia y la reconciliación, si se quiere realmente convertir a las escuelas en territorios de paz.

En los cuatro colegios investigados, la mayoría está de acuerdo en que el manual de convivencia y el mismo libro de seguimiento escolar contribuyen a mejorar el ambiente institucional y a controlar los brotes de violencia que puedan presentarse; sin embargo también coinciden en que los programas o planes que se diseñan para intervenir y atender estos escasos problemas deben conservar como principio rector, el respeto por las normas sociales y de convivencia entre los seres humanos.

Este trabajo, finalmente, se propone como el inicio de un proyecto de sensibilización y compromiso que tendrán que adelantar en un futuro no muy lejano todos los estamentos del estado que tienen la obligación de cuidar y educar a la juventud colombiana. La violencia que ha sufrido nuestro país desde tiempos remotos, se ha traslado y está aumentando en los momentos actuales dentro de los colegios. Por eso urge el diseño y aplicación de políticas humanas que impacten en toda la comunidad educativa y genere los cambios que la sociedad necesita para vivir en paz.

\section{Referencias}

Blaya, C., Derbarbieux, E., Del rey, R. y Ortega, R. (2002). Clima y violencia escolar un estudio comparativo entre España y Francia. Revista de la Educación, 339: 293-315.

Buss, A.H. y Perry, M.P. (1992). The aggression questionnaire. Journal ofPersonality and Social Psychology, 63: 452-459.

Campo, A.; Fernández, A. y Grisaleña, J. (2005). La convivencia en los centros de secundaria: un estudio de casos. Revista Iberoamericana de Educación, (38): 121-145.

Carpintero E., López F., Del Campo A., Soriano S. y Lázaro S. (2007). El bienestar personal y social de los adolescentes y la prevención del malestar y la violencia: Presentación y validación de un programa educativo. Revista de Investigación en Psicología, 10 (2): 29-41.

Chaux, E. (2002). Buscando pistas para prevenir la violencia urbana en Colombia: conflictos y agresión entre niños (as) y adolescentes en Bogotá. Estudios Sociales, 12: 43-53.

De la Villa, M. (2005). Actitudes socioconstruidas ante la violencia bullying en estudiantes de secundaria. Anuario de Psicología, 36(1), 61-81.

Del Rey, R. y Ortega, R. (2001). Programas para la prevención de la violencia escolar en España: la respuesta de las comunidades autónomas. Revista Interuniversitaria de Formación del Profesorado, (41): 133-145.

Díaz, A. (2005). Por qué se produce la violencia escolar y cómo prevenirla. Revista Iberoamericana de Educación, 37, 17-47

Díaz, M. y Aguado, J. (2005). La violencia entre iguales en la adolescencia y su prevención desde la escuela. Psicothema, 17(4), 549-558.

Domínguez, F. y Manzo M. (2011). Las manifestaciones del Bullying en adolescentes. Uaricha. Revista de Psicología (Nueva Época), 8 (17): 19-33.

Félix, F.; Soriano, M.; Godoy, C. y Martínez, I. (2008). Prevención de la violencia y promoción de la convivencia escolar en la Comunitat 
Valenciana (Plan PREVI). Aula Abierta,36(1-2), 97-110.

Górriz, A. (2009). Roles implicados en el acoso escolar: Comprensión de la mente, maquiavelismo y evitación de responsabilidad. Tesis Doctoral inédita. Valencia: Universitat Jaume I. Disponible en http://www.tdx. cat/bitstream/handle/10803/10530/gorriz. pdf? sequence $=1$

Hoyos, O.; Aparicio, J. y Córdoba, P. (2005). Caracterización del maltrato entre iguales en una muestra de colegios de Barranquilla Colombia. Psicología desde el Caribe, 16: 1-28.

Monjas y Avilés (2006). Programa de sensibilización contra el maltrato entre iguales. Valladolid: Consejería de Educación. Junta de Castilla y León.

Oliveros, M., Figueroa, L., Mayorga, G., Cano, G., Quispe, Y. y Barrientos, A. (2009). Intimidación en colegios estatales de secundaria del Perú. Revista Peruana de Pediatría,62: 68-78.

Olweus, D. (1998). Conductas de acoso y amenaza entre escolares. Madrid: Morata

Ortega R. y Del Rey, R. (2006). La mediación escolar en el marco de la construcción de la convivencia y la prevención de la violencia. Avances en supervisión Educativa, 2. Disponible en https://avances.adide.org/index.php/ase/ article/view/202

Ortega, R. y Mora, J. A. (1998). Violencia escolar. El problema del maltrato entre iguales. Cuadernos de Pedagogía, 270: 46-50

Paredes, M.T.; Álvarez, M.; Lega, L. y Vernon, A. (2008). Estudio exploratorio sobre el fenómeno del "bullying" en la ciudad de Cali, Colombia. Revista Latinoamericana de Ciencias Sociales, Niñez y Juventud, 6 (1): 295-317.

Paredes, M.T.; Lega, L.; Cabezas, H.; Ortega, M.; Medina, Y. y Vega, C. (2011). Diferencias transculturales en la manifestación del Bullying en estudiantes de Escuela Secundaria. Revista Latinoamericana de Ciencias Sociales, Niñez y Juventud, 9(2): 761-768.

Puglisi, B. (2012). La escuela como escenarios en los que se producen y reproducen violencias contra niños, niñas y adolescentes. Caracas: Instituto Latinoamericano de Investigaciones Sociales (ILDIS).

Ramos, C, Nieto, A. y Chaux, E. (2007). Aulas en Paz. Revista Interamericana de Educación para la Democracia, 1(1): 36-56. Disponible en https://www.mineducacion.gov.co/cvn/1665/ articles-164318_pdf_1.pdf

Trianes, M. V. (2000). La violencia en contextos escolares. Málaga: Aljibe. 